\title{
Bioanalysis
}

\section{Biomarker measurements: how far have we come and where are we heading?}

\author{
"...for successful translation of biomarkers into the clinic, it is \\ imperative that biomarker researchers do not approach their work \\ either with only biological or analytical aspects in mind..."
}

First draft submitted: 4 October 2016; Accepted for publication: 11 October 2016; Published online: 18 November 2016

Keywords: bioanalytical guidance $\bullet$ biomarker measurement challenges $\bullet$ biomarker qualification $\bullet$ biomarkers $\bullet$ fit-for-purpose validation $\bullet$ preanalytic factors $\bullet$ protein calibrators $\bullet$ sensitive technologies

Biomarker research has attracted the attention of scientists, clinicians, academicians, regulators and many others for at least two decades now, due to the compelling promise and the immense potential for use of biomarkers as diagnostic, prognostic and theranostic tools at the patient level and their use in drug development. While there have been sporadic successes, the translation of research into biomarkers that can be applied in the clinic is unrealized to a large extent, even today. In this editorial, the author shares a few thoughts and perspectives on the challenges related to measurement of biomarkers and where the field is heading.

By definition, 'biomarker' is a very broad term as it indicates a marker that can be objectively measured that reflects a biological process in vivo. Therefore, the relevant biomarkers to measure either for the purpose of drug development or for application at the patient level in the clinic, can come in a wide variety of forms. A vast range of technologies are used to measure the variety of biomarkers, from fluid biomarkers, cellular biomarkers, tissuebased biomarkers, imaging biomarkers and other physiological measures. For the purpose of this editorial, the discussion is focused on fluid biomarkers and to a large extent on protein biomarkers unless otherwise stated. However, some of the same principles tend to apply for biomarkers of other categories measured using various technologies.

\section{The biological processes \&} verification challenges: the chicken $\&$ the egg situation

When it comes to the discovery and implementation of biomarkers, there are two major aspects that the researchers engaged in biomarker work need to fully appreciate in order for us to move the field rapidly to realize their promise. The researchers engaged in this work need to appreciate both the biological and analytical validation aspects of the work, as both of these aspects are interrelated. Unless a specific, accurate and precise assay is available to measure the biomarker, the biological process to validate the biomarker may not be verified accurately. On the other hand, in the initial stages of biomarker research and development it is neither feasible nor practical to treat every biomarker as a definitive marker used for decision making at a patient level. To apply one uniform standard to every biomarker under investigation irrespective of the biological state of validation would become not only prohibitive due to significant investment of resources, time and money but is also not necessary as biological hypothesis may not yet have significant evidence. Consequently, biomarker discovery and development is an iterative process and biomarker selection in the early stages calls for scientific savvy on the part of the researcher, as the field, is often wide open and many different paths available to choose. Several regulatory agencies including the US

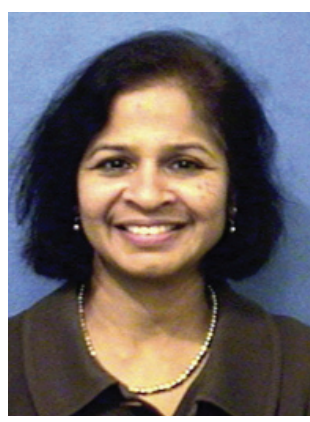

Lakshmi Amaravadi Translational Medicine \& Early Development, Sanofi-Genzyme, Framingham, MA, USA Lakshmi.Amaravadi@sanofi.com

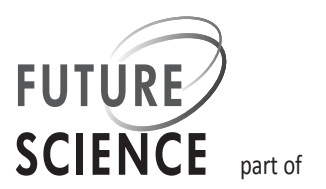


FDA's critical path initiative have been established to facilitate biomarker qualification and beginning to show progress [1-3]. To aid in this process of biomarker qualification, a fit-for-purpose or tiered approach to biomarker assay development and validation have been proposed and discussed at various scientific conferences and via publications [4-6]. The essential concepts of this approach provide a framework that allows for the iterative process of biomarker discovery and verification to progress in a step-wise manner, via the application of scientifically valid assays, ultimately with the biomarker reaching the highest level of biological and analytical validation required for decision making in clinical practice.

\section{Analytical challenges related to biomarkers}

Although it is tempting to categorize measurement of biomarkers in the same vein as the bioanalysis of other analytes (e.g., measurement of drugs and therapeutics), in reality due to the nature of the biomarkers that are endogenously produced and owing to their high degree of heterogeneity, the calibrator material used does not truly represent the endogenous biomarker. Due to the number of factors that contribute to the variability of biomarkers (including inter- and intraindividual variability over time) it is important to appreciate and consider all of these factors while developing and validating the biomarker assays to measure them, as well as consider the impact of all of these factors when analyzing the data. Numerous authors have discussed the analytical challenges related to biomarkers and proposed a variety of approaches to categorize biomarker measurements $[4,7]$. Despite this, when the FDA has incorporated a brief section on measurement of biomarkers in the bioanalytical method validation draft guidance $[8]$, a heated discussion ensued within the scientific community that was later followed by a separate conference dedicated to the discussion on biomarker measurements and related challenges [9,10]. At this year's Crystal City VI conference [10], biomarker measurements and challenges were discussed from immunoassay and LC-MS/MS assay perspectives and several case studies of success and issues identified were discussed. The conference report covers the details of the discussion [10]. While this is the beginning of the discussion in this field, it is by no means the end in my perspective, as there is no one size fits-all solution.

The primary analytical challenges related to biomarker measurements still remain such as:

- Specificity: lack of specific reagents and other issues can lead to potentially misleading results;

- Sensitivity: molecules of high potency that are typically present in very low levels may not be measurable;
- Stability: often times the biomarkers of interest tend to be labile and significant investment may be needed to study this parameter upfront, and ensure stability in clinical settings to yield meaningful results.

Merits and drawbacks of ligand-binding assays and/ or immunoaffinity LC-MS hybrid methods have been discussed extensively [11,12]. The single most important challenge with measurement of protein biomarkers still continues to be the availability of representative reference material. The protein standard material during early phases of drug development is obtained from a variety of sources and spiked into matrix and used as a calibrator to measure endogenous biomarker in samples of interest. However, both the matrix used for the calibrator and the sample matrix are likely to contain endogenous biomarker that confounds the measurement (unlike in the drug concentration assays where the reference standard is also often the analyte of interest). The endogenous biomarker levels may vary between individual matrices as well as over time in the matrix drawn from the same individual. In addition, due to differences in the properties of endogenous versus recombinant biomarker used as calibrator material, the accuracy of measurement is often times questionable and may represent relative accuracy. In addition, parallelism is a parameter that needs be assessed and understood in these assays in order to ensure that the calibrator utilized represents the endogenous biomarker of interest, as parallelism is the primary indicator of similarity between analyte and the calibrator in ligand-binding assays.

While most analytical scientists understand the concept of analyte stability, it should be noted that in the case of protein biomarker measurement, because of the differences in the recombinant calibrator material used versus endogenous biomarker, stability of the biomarker should be assessed using samples containing endogenous material and not the calibrator spiked samples. There have been examples in the literature where use of calibrator spiked samples lead to misleading results [13] that could potentially lead to erroneous decisions if not recognized.

Significant amount of published biomarker research by various researchers involves the use of commercial kits that are intended for research use only. The calibrator materials often are not fully characterized in these kits and it is also not uncommon to find that the reagents used are also not fully characterized. This may lead to nonreproducible results from lot-to-lot. Therefore, it is imperative that the scientists involved in biomarker research and development understand how to use the kits, how to characterize the reagents involved, so that a 
valid assay can be developed to suit the question at hand. This approach allows for development of scientifically valid assays that can lead to appropriate decision making based on stage of development and best use of resources. Although this article cannot cover all aspects of this in detail, it is important to note that considerations also vary significantly for multiplex biomarker assays versus single biomarker assays [14].

\section{The preanalytic factors}

Another major factor that is neither biological nor analytical but has a huge impact on biomarker measurement is the issue of biological sample collection variables and stability.

These factors that broadly fall under logistical challenges tend to be underestimated or even neglected, however, they can have a huge impact on the final result, irrespective of the type of sample collected [15]. Often, the scientists involved in biomarker discovery and development are far removed from the clinical sites where the samples are collected from patients. There are numerous variables starting from collection of appropriate samples (in the appropriate manner by the technicians at the clinical site), to storage during transit (often may involve $-20 \mathrm{C}$ or $-80 \mathrm{C}$ or even LN2 storage), shipping and storage at the research laboratory can impact the results. The majority of these variables can impact the stability of sample/biomarker of interest and it is not uncommon that a significant amount of time may be spent in investigating biomarkers of sample stability, if the sample logistics have not been taken care of and tracked appropriately.

\section{Where are we heading?}

With the availability of newer technology platforms, researchers now have access to ultrasensitive and specific technology solutions, e.g., Simoa ${ }^{\mathrm{TM}}$, SMC $\mathrm{CM}^{\mathrm{TM}}$ that can allow measurement at single molecule level

\section{References}

1 Amur S, Frueh FW, Lesko LJ, Huang SM. Integration and use of biomarkers in drug development, regulation and clinical practice: a US regulatory perspective. Biomark. Med. 2(3). 305-311 (2008).

2 Amur SG, Sanyal S, Chakravarty AG et al. Building a roadmap to biomarker qualification: challenges and opportunities. Biomark. Med. 9(11), 1095-1105 (2015).

3 Wagner JA, Ball JR. Implications of the institute of medicine report: evaluation of biomarkers and surrogate endpoints in chronic disease. Clin. Pharmacol. Ther. 98(1), 12-15 (2015).

4 Lee JW, Devanarayan V, Barrett YC et al. Fit-for-purpose method development and validation for successful biomarker measurement. Pharm. Res. 23(2), 312-328 (2006). and immunoaffinity LC-MS/MS approaches [16,17]. However, the measurement of biomarkers is a complex problem that involves consideration of multiple factors and, therefore, calls for an integrated approach that involves, an understanding of the issues, the biological/clinical context, in order to be able to implement the right solutions for answering the questions in hand. While it would be simpler to treat every biomarker the same and apply one uniform analytical validation criteria, it is important for us to note that this approach would require a biomarker calibrator to be developed to a level such that it truly represents the endogenous version from an analytical perspective. However, it should be understood that in majority of the cases a biomarker is developed in an iterative process using a tiered approach that involves application of scientifically valid assays as mentioned above, ultimately reaching a standard suitable for application at patient level for clinical decision making. Therefore, for successful translation of biomarkers into the clinic, it is imperative that biomarker researchers do not approach their work either with only biological or analytical aspects in mind, but appreciate how these two aspects together influence the output of the biomarker assays. Such a translational approach and mindset to application of biomarkers can significantly advance the application of biomarkers in drug development and in the clinic.

\section{Financial \& competing interests disclosure}

The author has no relevant affiliations or financial involvement with any organization or entity with a financial interest in or financial conflict with the subject matter or materials discussed in the manuscript. This includes employment, consultancies, honoraria, stock ownership or options, expert testimony, grants or patents received or pending, or royalties.

No writing assistance was utilized in the production of this manuscript.
5 Lee JW. Method validation and application of protein biomarkers: basic similarities and differences from biotherapeutics. Bioanalysis 1(8), 1461-1474 (2009).

6 Timmerman P, Lowes S, McDougall S et al. Scientific or regulated validation: a tiered approach? Meeting report from a joint EBF/DVDMDG workshop. Bioanalysis 7(14), 1703-1710 (2015).

7 Houghton R, Chamberlain J. Conference report: analytical challenges in the qualification and validation of pharmacodynamics biomarkers. Bioanalysis 3(9), 945-948 (2011).

8 US FDA, US Department of Health and Human Services, draft guidance for industry; bioanalytical method validation (revised) (2013). www.fda.gov/downloads/Drugs/ GuidanceRegulatoryInformation/Guidance/UCM368107.pdf 
9 Booth B, Arnold ME, DeSilva B et al. Workshop report: Crystal City V-quantitative bioanalytical method validation and implementation: the 2013 revised FDA guidance. AAPS J. 17(2), 277-288 (2015).

10 Arnold ME, Booth B, King L, Ray C Workshop report: Crystal City VI-bioanalytical method validation for biomarkers. AAPS J. doi:10.1208/s12248-016-9946-6 (2016) (Epub ahead of print).

11 Miller RA, Spellman DS. Mass spectrometry-based biomarkers in drug development. Adv. Exp. Med. Biol. 80, 341-359 (2014).

12 Ackermann B, Neubert H, Hughes $\mathrm{N}$ et al. White paper on recent issues in bioanalysis: focus on new technologies and biomarkers (Part 2 - hybrid LBA/LCMS and input from regulatory agencies). Bioanalysis 7(23), 3019-3034 (2015).

13 Fraser S, Fleener C, Ogborne K, Soderstrom C When close is not close enough: a comparison of endogenous and recombinant biomarker stability samples. Bioanalysis $7(11)$, 1355-1360 (2015).

14 Jani D, Allinson J, Berisha F et al. Recommendations for use and fit-for-purpose validation of biomarker multiplex ligand binding assays in drug development. AAPS J. 18(1), 1-14 (2016).

15 Hewitt SM, Badve SS, True LD. Impact of preanalytic factors on the design and application of integral biomarkers for directing patient therapy. Clin. Cancer Res. 18(6), 1524-1530 (2012).

16 Yeung D, Ciotti S, Purushothama S et al. Evaluation of highly sensitive immunoassay technologies for quantitative measurements of sub-pg/ml levels of cytokines in human serum. J. Immunol. Methods 437, 53-63 (2016).

17 Ackermann BL. Immunoaffinity MS: adding increased value through hybrid methods. Bioanalysis 8(15), 1535-1537 (2016). 